\title{
Stem Cells, Cancer and the Theory of Cancer Stem Cells in Solid Tumours
}

\author{
Abdulmajeed Almutary* \\ Medical Biotechnology, Flinders University, South Australia
}

*Corresponding author: Abdulmajeed Almutary, Medical Biotechnology, College of Medicine and Public Health, Flinders University, GPO Box 2100, Adelaide 5001, South Australia

Submission: 監 March 06, 2018; Published: 眥 March 22, 2018

\begin{abstract}
Solid tumours are an immense cancer burden and a main therapeutic challenge. The theory of cancer stem cells provides an attractive cellular mechanism to account for the therapeutic resistance exhibited by many of these tumours. There is strong evidence that various solid tumours are hierarchically managed and sustained by notable subpopulations of cancer stem cells. Recent evidence of cancer stem cells emerged from a mouse model epithelial tumorigenesis, also some models of heterogeneity may apply. Haematopoietic stem cells (HSCs) have the capability to renew themselves and produce lineages of the blood; yet the signals that control HSc self-renewal remain indistinct. WnT signalling pathway has crucial role in the expression of activated $\beta$-catenin which expands the HSCs in a long-term cultures. In addition, Wnt signalling can interrupt the lymphocyte progenitor cells proliferation and affect the cells development. Other similar signalling pathways such as Notch and Hedgehog (Hh) are found in normal stem cells. Therapeutic targeting of cancer stem cells and tumour population could shed light on how to supress tumour growth. In this review, we suggest that by developing a new anti-cancer stem cell therapeutic agent that targets embryonic signalling pathways of cancer stem cells can improve the treatment of the disease.
\end{abstract}

Keywords: Stem cells; Cancer cells; Cancer stem cells; Self-renewal; Oncogenesis; Leukaemogenesis; Progenitor cells

\section{Background}

Cancer originally develops from normal cells that have the ability to proliferate irregularly and turn into malignant. These cancerous cells then grow clonally into tumours and have the ability to metastasize. A crucial question in cancer biology is which cells can be transformed to form tumours [1]. Recent studies showed that the presence of cancer stem cells have the ability to regenerate tumours [2]. These cancer stem cells have many characteristics similar with normal stem cells, including selfrenewal and differentiation [2]. With increasing the number of evidence that cancer stem cells exist in a wide range of tumours, it is becoming important to understand the molecular mechanism that regulate self-renewal and differentiation because corruption of genes involved in these pathways likely participates in tumour growth [3].

Haematopoietic stem cells (HSCs) have been isolated from humans and mice and have been involved in the generation and regeneration of the blood-forming and immune systems [4]. Stems cells isolated from variety of organs might have the potential to be used for therapy in the near future [5]. The recent discovery that bone marrow and HSCs are able to rise non-haematopoietic tissues proposed that these cells may have better differentiation potential than was presumed previously. Experiments are needed to determine whether the cells from the bone marrow that have the capability to increase different non-haematopoietic lineages are actually HSCs or another population [6]. This study focuses on three aspects of the relationship between stem cells and tumour. Firstly, the similarities observed in the mechanisms that control self-renewal of normal cells and cancer cells. Secondly, the potential that tumour cells may emerge from normal stem cells. Lastly, the concept that tumours might contain unique cells with indefinite proliferative that control the formation and growth of tumour.

\section{Self-Renewal of Haematopoietic Stem Cells}

One of the substantial issues in stem cell biology is understanding the mechanisms that maintain self-renewal. Self-renewal is important to stem cell function, because it is demanded by many types of stem cells to persist for the lifetime of the animal. Although, stem cells from different organs may vary in their development potential, each stem cell must self-renew and regulate the relative balance between self-renewal and differentiation. Fathoming the regulation of normal stem cells self-renewal is also fundamental to understanding the regulation of cancer cell proliferation. This is because cancer can considered being a disease of unregulated selfrenewal [7]. 
In the haematopoietic system, stem cells are heterogeneous with their ability to self-renew [8]. Multipotent progenitors contain $0.05 \%$ of mouse bone-marrow cells, and can be separated into three different populations: short-term self-renewing HSCs, longterm self-renewing and multipotent progenitors without detectable self-renewal potential. These populations structure a lineage in which the long-term HSCs increase short-term HSCs, which leads to raise multipotent progenitor [9]. Although long-term HSCs produce mature haematopoietic cells for the lifetime of mouse, multipotent progenitors and short-term HSCs reconstitute lethally irradiated mice for less than eight weeks [10]. Despite the fact that HSCs functional properties and phenotypic have been extensively characterised, the substantial question is how self-renewal regulate still remains unanswered. In majority of cases, combination of growth factors that can stimulate potent proliferation cannot prevent the differentiation of HSCs in long-term cultures. Although attempts have been made in identifying culture conditions that maintain HSC activity, it has demonstrated exceedingly difficult to identify combinations of defined growth factors that can lead to a significant expansion in the number of progenitors with transplantable HSC activity [11]

\section{Pathways that Controls Stem Cell Self-Renewal and Oncogenesis}

Evidence shows that many pathways that are associated with cancer may also control normal cell development [12]. For instance, the prevention of apoptosis by compelling the expression of the oncogene bcl-2 results in expansion of HSCs in vivo. This suggests that cell death has a function in regulating the homeostasis of HSCs. Notch, Sonic hedgehog (Shh) and Wnt signalling pathways may also regulate stem cell self-renewal [13]. Notch activation in HSCs in culture by the ligand Jasgged-1 have continually increased the amount of primitive progenitor activity that can be noticed in vitro and in vivo, this indicates that Notch activation promotes HSc self-renewal, or at least the maintenance of multi-potentiality. Shh signalling has also been involved in the regulation of self-renewal by the discovery that populations highly enriched for human HSCs showed increase self-renewal in response to shh stimulation in vitro [14].

The influence of Notch and Shh in the self-renewal of HSCs is especially interesting in the studies that implicate the pathways in the control of self-renewal of stem cells [13]. Another interesting pathway that has also involved in the regulation of both self-renewal and oncogenesis in different organs is the Wnt signalling pathway. Wnt proteins are intercellular molecules that can coordinate the development of several organisms and lead to cancer when dysregulated. The discovery of Wnt proteins in bone marrow suggests that they may interact with HSCs as well [15]. In a recent study used highly purified mouse bone-marrow HSCs showed that over expression of activated b-catenin in long-term cultures of HSCs contributed to the expansion of transplantable HSCs identified by both phenotype and function [16].
In addition, ectopic expression of Axin which is an inhibitor of Wnt signalling has led to the inhibition of HSC proliferation, increased in vitro death of HSCs and reduced reconstitution in vivo (Ross et al., 2000). Other studies have shown that soluble Wht proteins from supernatants influenced the proliferation of haematopoietic progenitors from human bone marrow and mouse fetal liver [17]. Epidermal and gut progenitor studies suggest that Wnt signalling may lead to the regulation of stem cells/progenitors cell self-renewal in some tissues. Human keratinocytes cultures with higher proliferative have shown increased levels of b-catenin in contrast with keratinocytes with lower proliferative capacity. Also, retroviral transduction of activated b-catenin found to increase epidermal stem cell self-renewal and decreased differentiation [18]. Data from in vivo transgenic mice reveals that the activation of Wnt signalling pathway in epidermal stem cells leads to epithelial cancers [19]. Moreover, mice lacking TCF-4 which is one of the transcriptional mediators of the Wnt signalling pathway quickly used the undifferentiated progenitors in the crypts of the gut epithelium throughout fetal development [20]. This suggests that this pathway is required for the maintenance of self-renewal of gut epithelial stem cells. Therefore, the above findings demonstrate that Wnt signalling may enhance stem cell self-renewal on diverse epithelia in addition to HSCs. However, the molecular mechanisms by which Wnt signalling interrupt stem cells remain unclear also the involvement of Wnt, Notch and Shh pathways control the stem and progenitor cell self-renewal [19].

\section{Leukaemogenesis and Self-Renewal}

If presumably that the signalling pathways normally control stem cells self-renewal can lead to tumorigenesis when dysregulated, then stem cells themselves are the target of transformation in some types of cancer. There are two reasons to support this case. One is because stem cells ability to self-renewal already activated and maintaining this activation could be simpler than turning to more differentiated cell that is why fewer mutations may be needed to maintain self-renewal than to activate it ectopically [20]. Secondly, by self-renewing, stem cells persist for a longer periods of time, rather than dying after short period of time just like many mature cells in highly proliferative tissues. Thus, this means there is a greater chance for mutation to accumulate in each single stem cell than in most mature cell types [21].

Even some restricted progenitor cells are less expected than stem cells to undergo neoplastic transformation due to shorter proliferative period of time before terminally differentiating. Restricted haematopoietic progenitors of myeloid and lymphoid lineages were unsuccessful to self-renewal detectably on transplantation [22]. As a result of this, restricted progenitors would first need to obtain the extensive self-renewal potential of stem cells to have the chance to experience additional mutations that leads to transformation [23]. Even thought, restricted progenitors could possibly be transformed either by obtaining mutations that drives them to self-renewal like stem cells, or by inheriting a present mutations from stem cells [24]. 


\section{Targeting Mutations in Stem Cells}

In most of cancers, the target cell of transforming mutations cannot be identified, however, there is considerable evidence that some types of leukaemia emerge from mutations that accumulate in HSCs. The

cells are able of initiating human acute myeloid leukaemia (AML) in non-obese diabetic mice that have a CD34+ CD38phenotype in most AmL subtypes which similar phenotype found in normal HSCs [25]. On the contrary, CD34+ CD38- leukaemia cells are not able to transfer disease to mice in the vast majority of cases, although they exhibit a leukemic blast phenotype (Lobo et al., 2007). This indicates that normal HSCs rather than committed progenitors are the biggest for leukemic transformation [26]. The most persistent chromosomal abnormalities in AML includes the 8;21 translocation which leads in AML1-ETO chimeric transcripts in leukemic cells. From patients in remission, the AML1-ETO transcript was identified in a fraction of normal HSCs in the marrow [27]. These prospectively isolated HSCs were not leukemic and are able to differentiate to normal myeloerythroid cells in vitro. This means that the translocation happened originally in normal HSCs and those extra mutations in part of these HSCs or their progeny lead to leukemic. Studies suggested the normal HSCs were CD34+ CD38- Thy-1+, while the leukemic blasts were CD34+ CD38-Thy-1Despite the fact that the translocation must have occurred in the normal HSCs, a following transforming mutation might have taken place [28]. This is dependent on the consequence of neoplastic proliferation and it was the loss of Thy- 1 expression. The concept that stem cells are a common target of leukemic transformation or pre-leukemic events is also propped by lymphoid and chromic myeloid leukaemia in which clonotypic leukaemia - related chromosomal rearrangement have also been discovered in CD34+ CD38- cells. Therefore, divers of leukaemia may originate from mutations that accumulate in HSCs to cause their malignant transformation at the stage of stem cells or at their progeny [29].

\section{Progenitor Cells Used as a Target of Transformation}

Despite the fact that stem cells are mostly the largest genetic events that are important or sufficient for malignant transformation, in other situations restricted progenitors or even differentiated cells may become transformed [2]. When targeting the expression of transgenes specifically to restricted myeloid progenitors by using the hMRP-8 promoter, it is likely to create a mouse model in which myeloid leukaemia emerges from restricted progenitors [30]. These leukaemias resemble human leukaemias in many points, even when the targeted genetic changes led the leukaemias to increase from originate restricted progenitors rather than stem cells. For instance, a study generated transgenic mouse models for myeloid leukaemias using an hMRP-8 promoter, that can targets the expression of transgenes specifically to myeloid progenitors. The identification of anti-apoptotic gene bcl-2 in the myeloid lineage can cause a disease in mice that is identical to human chromic myelomonocytic leukaemia such as monocytosis and neutropenia; however, mice rarely develop acute malignancies [31].
To examine whether extra mutations are required to synergize with bcl-2 enhance AML, hMRP8-bcl-2 transgenic mice were bred with with Ipr/Ipr fas-deficient mice. The lack of these two apoptosis pathways caused AML in 15\% of the mice [32]. Myeloblasts expansion was found in haematopoietic tissues of mice, with highly lowered number of granulocytes in the bone marrow and blood [33]. Studies have shown that prevention of cell death is an important event in myeloid leukaemogenesis and that restricted progenitors can be transformed. As previously mentioned in the case of emerging human leukaemias it is mostly that stem cells accumulate the mutations that are essential for neoplastic proliferation; however, these mutations may gather in stem cells even while the effects of the mutations are explicated in restricted progenitors. Especially mutations that accumulate in stem cells may lead to neoplastic proliferation of primal progenitors downstream of stem cells [17].

\section{Evidence of Cancer in Stem Cells}

It was firstly documented for leukaemia and multiple myeloma that a small group of cancer cells were able to extend proliferation [34]. For instance, when mouse myeloma cells were isolated from mouse ascites and seeded in clonal in vitro colony-forming assays, there was only 1 in 10, 1000 to 1 in 100 cancer cells formed colonies. Even after leukaemic cells were transplanted in vivo, just $1-4 \%$ of cells managed to form spleen colonies. Due to the differences in clonogenicity among the leukaemia cells, this has reflected the differences in clonogenicity among normal haematopoietic cells [35].

Two formal possibilities are to explain the differences in colony formation, one is either all leukaemia cells had a low probability of proliferating largely in these assays such that all leukaemia cells had the ability to act as leukaemic stems cells, or majority of leukaemia cells were incapable to proliferate and only small a small group of cells was consistently clonogenic [35]. The second possibility might be proved by separating different classes of leukaemia cells and identifying if one subset is highly enrich for clonogenic capacity while the other cells are depleted from clonogenicity [35-37].

\section{Conclusion}

Solid tumours emerge in organs that acquire stem cell populations. The tumours in these tissues are made of heterogeneous populations of cancer cells that show variable signs in their ability to proliferate and form new tumours. In both central nervous system and breast cancers tumours, cancer cells shown different ability develop regenerate tumours. Although many of the cancer cells have the capabilities to divide, some of the cancer stem cells that has the unique ability to intensively proliferate and form new tumours can be recognized based on marker expression. Increasing evidence suggest that the pathways control the selfrenewal of the normal cells are disrupted in cancer cells resulting in the persistence expansion of self-renewal cancer cells and tumour formation. This study suggests that specified agents for the defective self-renewal pathways in cancer cells could be able to improve the outcomes in the treatment of these diseases. 


\section{Acknowledgment}

A special thanks to Shamez Talib for supporting, inspiring and encouraging me throughout my $\mathrm{PhD}$ study, related research and publications.

\section{References}

1. Bergers G, Benjamin IE (2003) Tumorigenesis and the angiogenic switch. Nat Rev Cancer 3(6): 401-410.

2. Timaner M, Letko Khait N, Kotsofruk R, Benguigui M, Beyar katz O, et al. (2018) Therapy-educated mesenchymal stem cells enrich for tumor initiating cells. Cancer Res pii: canres.1547.2017.

3. Bakhshinyan D, Adile AA, Qazi MA, Singh M, Kameda smith MM, et al (2018) Introduction to cancer stem cells: past, present, and future. Can cer stem cells. Methods Mol Biol 1692: 1-16.

4. Ivanova NB, Dimos JT, Schaniel C, Hackney JA, Moore KA, et al. (2002) A stem cell molecular signature. Science 298(5593): 601-604.

5. Lanza R, Gearhart J, Hogan B, Melton D, Pedersen R, et al. (2005) Essentials of stem cell biology. Academic press, USA.

6. Holland AM, Góñez LJ, Harrison LC (2004) Progenitor cells in the adult pancreas. Diabetes/metabolism research and reviews 20(1): 13-27.

7. Morrison SJ, Shah NM, Anderson DJ (1997) Regulatory mechanisms in stem cell biology. Cell 88(3): 287-298.

8. Alhajj M, Clarke MF (2004) Self renewal and solid tumor stem cells. Oncogene 23(43): 7274-7282.

9. Sacchetti B, Funari A, Michienzi S, Di Cesare S, Piersanti S, et al. (2007) Self-renewing osteoprogenitors in bone marrow sinusoids can organize a hematopoietic microenvironment. Cell 131(2): 324-336.

10. Moore KA, Lemischka IR (2006) Stem cells and their niches. Science 311(5769): 1880-1885.

11. Rehman J (2010) Empowering self-renewal and differentiation: the role of mitochondria in stem cells. J Mol Med (Berl) 88(10): 981-986.

12. Hartwell 1H, Kastan MB (1994) Cell cycle control and cancer. Science-aaas-weekly paper edition 266(5192): 1821-1828

13. Valk lingbeek ME, Bruggeman SW, Van Lohuizen M (2004) Stem cells and cancer: the polycomb connection. Cell 118(4): 409-418.

14. Pajcini K, Speck N, Pear W (2011) Notch signaling in mammalian hematopoietic stem cells. Leukemia 25(10): 1525-1532.

15. Willert K, Brown JD, Danenberg E, Duncan AW, Weissman IL, et al. (2003) Wnt proteins are lipid-modified and can act as stem cell growth factors. Nature 423(6938): 448-452.

16. Nishino T, Osawa M, Iwama A (2012) New approaches to expand hematopoietic stem and progenitor cells. Expert opinion on biological therapy 12(6): 743-756.

17. Rossi DJ, Jamieson CH, Weissman IL (2008) Stems cells and the pathways to aging and cancer. Cell 132(4): 681-696.

18. Liu S, Dontu G, Wicha MS (2005) Mammary stem cells, self-renewal pathways, and carcinogenesis. Breast Cancer Res 7(3): 86-95.

19. Watt FM (2000) Epidermal stem cells as targets for gene transfer. Human gene therapy 11(16): 2261-2266
20. Korinek V, Barker N, Moerer P, Van Donselaar E, Huls G, et al. (1998) Depletion of epithelial stem-cell compartments in the small intestine of mice lacking tcf-4. Nat Genet 19(4): 379-383.

21. Li F, Tiede, Massagué J, Kang Y (2007) Beyond tumorigenesis: cancer stem cells in metastasis. Cell Res 17(1): 3-14.

22. Baserga R (1985) The biology of cell reproduction. Harvard university press, USA.

23. Vermeulen L, Sprick M, Kemper K, Stassi G, Medema J (2008) Cancer stem cells-old concepts, new insights. Cell death \& differentiation 15(6): 947-958.

24. Johe KK, Hazel TG, Muller T, Dugich Djordjevic MM, Mckay R (1996) Single factors direct the differentiation of stem cells from the fetal and adult central nervous system. Genes Dev 10(24): 3129-3140.

25. Pardal R, Clarke MF, Morrison SJ (2003) Applying the principles of stemcell biology to cancer. Nat Rev Cancer 3(12): 895-902.

26. Lobo NA, Shimono Y, Qian D, Clarke MF (2007) The biology of cancer stem cells. Annu Rev Cell Dev Biol 23: 675-699.

27. Miyamoto T, Weissman IL, Akashi K (2000) Aml1/eto-expressing nonleukemic stem cells in acute myelogenous leukemia with 8; 21 chromosomal translocation. Proc Natl Acad Sci U S A 97(13): 7521-7526.

28. Passegué E, Jamieson CH, Ailles LE, Weissman IL (2003) Normal and leukemic hematopoiesis: are leukemias a stem cell disorder or a reacquisition of stem cell characteristics? Proc Natl Acad Sci U S A 100(Suppl 1): $11842-11849$.

29. Blair A, Hogge D, Ailles l, Lansdorp P, Sutherland H (1997) Lack of expression of thy-1 (CD90) on acute myeloid leukemia cells with long-term proliferative ability in vitro and in vivo. Blood 89(9): 3104-3112.

30. Loaiza B, Rojas E, Valverde M (2012) The new model of carcinogenesis: the cancer stem cell hypothesis. Carcinogen.

31. Halsey C (2009) The role of gata-1 isoforms in haematopoiesis. University of Glasgow, UK.

32. Le Pogam C, Krief P, Beurlet S, Soulié A, Balitrand N, et al. (2013) Localization of the nras: bcl-2 complex determines anti-apoptotic features associated with progressive disease in myelodysplastic syndromes. Leuk Res 37(3): 312-319.

33. Traver D, Akashi K, Weissman IL, Lagasse E (1998) Mice defective in two apoptosis pathways in the myeloid lineage develop acute myeloblastic leukemia. Immunity 9(1): 47-57.

34. Ginestier C, Hur MH, Charafe Jauffret E, Monville F, Dutcher J, et al. (2007) Aldh1 is a marker of normal and malignant human mammary stem cells and a predictor of poor clinical outcome. Cell stem cell 1(5): 555-567.

35. Houghton J, Morozov A, Smirnova I, Wang TC (2007) Stem cells and cancer. Seminars in cancer biology. Elsevier 17(3): 191-203.

36. Reya T, Duncan AW, Ailles L, Domen J, Scherer DC, et al. (2003) A role for wnt signalling in self-renewal of haematopoietic stem cells. Nature 423(6938): 409-414.

37. Ross SE, Hemati N, Longo KA, Bennett CN, Lucas PC, et al. (2000) Inhibition of adipogenesis by wnt signaling. Science 289(5481): 950-953. 
Creative Commons Attribution 4.0 International License

For possible submissions Click Here

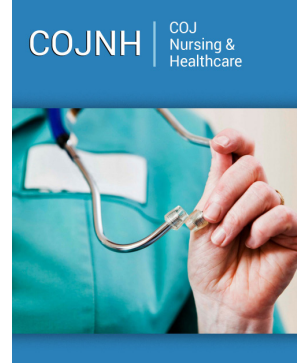

\section{COJ Nursing \& Healthcare}

Benefits of Publishing with us

- High-level peer review and editorial services

- Freely accessible online immediately upon publication

- Authors retain the copyright to their work

- Licensing it under a Creative Commons license

- Visibility through different online platforms 